Case Report

\title{
Intraoperative Visualization of Bilateral Thrombosis in the Posterior Inferior Cerebellar Artery Apparent in the Telovelomedullary Segment
}

\author{
Edin Nevzati, Bawarjan Schatlo, Ali-Reza Fathi, Javier Fandino, and Carl Muroi \\ Department of Neurosurgery, Kantonsspital Aarau, Tellstraße, 5001 Aarau, Switzerland \\ Correspondence should be addressed to Carl Muroi; carl.muroi@ksa.ch
}

Received 12 July 2014; Revised 15 September 2014; Accepted 15 September 2014; Published 28 September 2014

Academic Editor: Pablo Mir

Copyright (C) 2014 Edin Nevzati et al. This is an open access article distributed under the Creative Commons Attribution License, which permits unrestricted use, distribution, and reproduction in any medium, provided the original work is properly cited.

Unilateral posterior inferior cerebellar artery (PICA) thrombosis is frequent. However, bilateral PICA thrombosis is rare. Herein we report about an intraoperative visualization of a bilateral thrombosis of the telovelomedullary segment of the PICA. A 74-yearold woman was admitted to our department on day two of a bilateral PICA thrombosis with developing cerebellar infarction. Her Glasgow Coma Scale score dropped from 15 to 13, and cranial computed tomography revealed compression of the fourth ventricle with consecutive occlusive hydrocephalus. After the insertion of an external ventricular drainage, the patient underwent urgent suboccipital decompressive craniectomy with removal of infarcted cerebellar tonsils, which allowed the bilateral visualization of the thrombosed telovelomedullary segments. The surgical access may offer surgical therapeutic options in a hyperacute occlusion, such as thromb-/embolectomy or bypass procedures.

\section{Introduction}

Unilateral posterior inferior cerebellar artery (PICA) thrombosis is the most frequent type of cerebellar infarction and accounts for approximately $2 \%$ of all ischemic strokes [1]. Depending on the site of occlusion, clinical manifestation varies widely, from lack of symptoms to a severe medullary and cerebellar picture. Complete Wallenberg's syndrome is found in up to $15 \%$ of ischemic strokes in the PICA distribution [2]. Due to its small diameter (about 2.5 millimeters) [3], tomographic imaging of the PICA may be flawed by artifacts and limited by uncertainties. Therefore, in addition to the infarct distribution, angiography is the modality of choice for the diagnosis of occlusion. A macroscopic surgical visualization of bilateral acute PICA thrombosis has not been reported to date. Herein we report about an intraoperative visualization of a bilateral thrombosis of the telovelomedullary segment of the PICA. Surgical and clinical management strategies are briefly discussed.

\section{Case Report}

A 74-year-old woman was initially seen in the emergency department of a peripheral hospital presenting with acute onset of headache, dizziness, and limb weakness. Her medical history revealed cerebrovascular risk factors, that is, long year tobacco abuse, hypertension, and dyslipidemia. The patient was clearly conscious with a Glasgow Coma Scale (GCS) of 15 but had a left-sided Wallenberg's syndrome, right brachial weakness (M4), left central facial paresis, left lateral gaze nystagmus, dysarthria, bilateral dysmetria on finger-tonose and heel-to-shin tests, and truncal ataxia so severe that the patient could not stand. National Institute of Health Stroke Scale (NIHSS) score was 9. Initial cranial computed tomography (CT) did not show any signs of cerebellar pathologies. The patient was treated with aspirin and statin as an embolic infarction could not be ruled out. Electrocardiography showed intermittent atrial fibrillation with spontaneous conversion to sinus rhythm on the second day of 


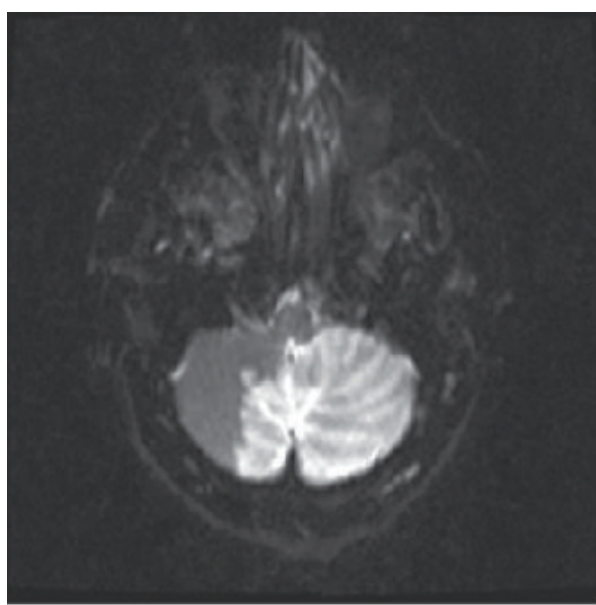

(a)

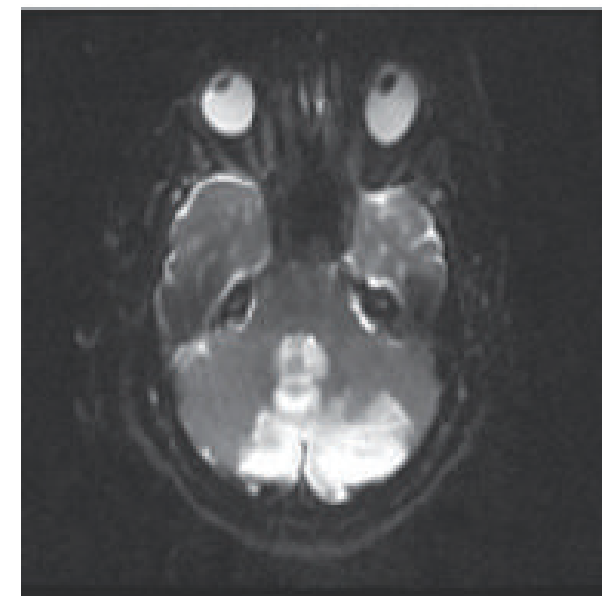

(b)

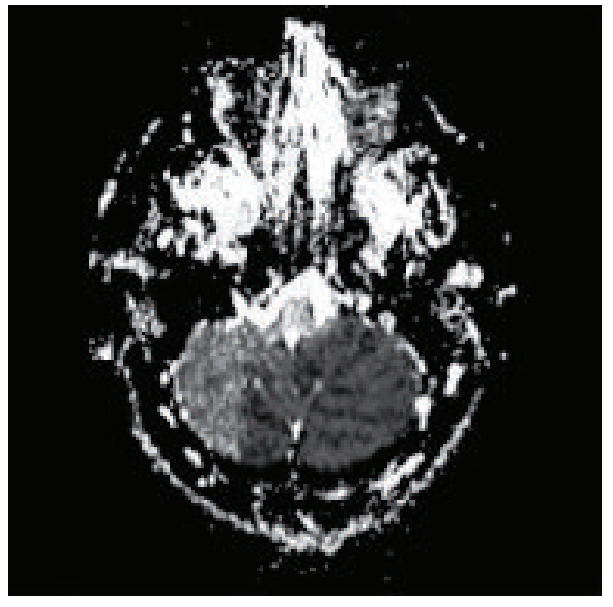

(c)

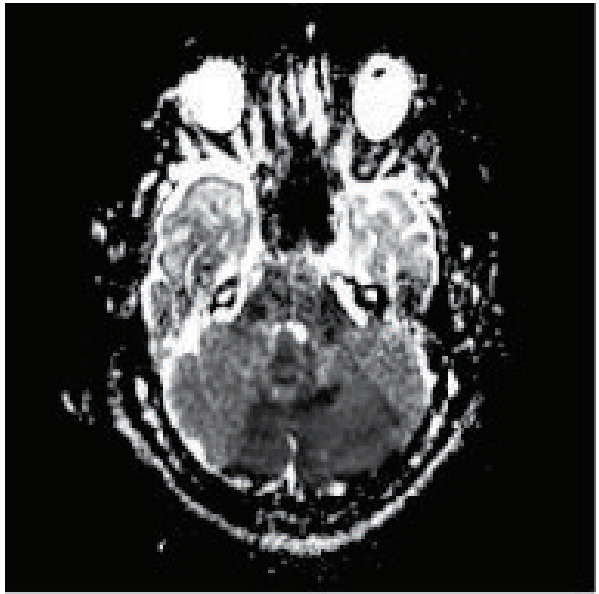

(d)

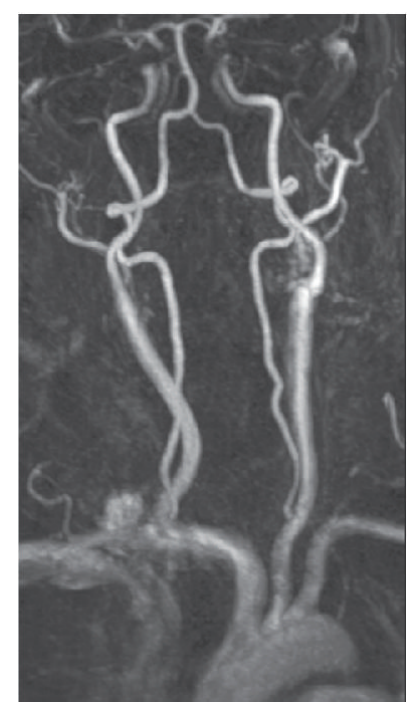

(e)

FIGURE 1: Cranial MRI performed on day two after bilateral PICA infarct. Diffusion-weighted images (a)-(b) and diffusion coefficient maps (c)-(d) revealing fresh infarcts. The 3-dimensional time-of-flight magnetic resonance angiogram shows patency of both vertebral arteries, whereas absence of flow-related signal in both inferior posterior cerebellar arteries is demonstrated (e).

hospitalization. Transthoracic echocardiography could not identify any atrial thrombi or valvular pathologies. On day two after onset, a magnetic resonance imaging (MRI) scan was performed. Diffusion-weighted imaging showed left lateromedullar hyperintensity as well as an infarction of both cerebellar tonsils (Figures 1(a)-1(d)). Moreover, the distribution of the bilateral PICA infarct corresponded to type B according to Kang et al. [4], with a complete infarct in the left inferior cerebellar hemisphere and a less extensive infarct in the right hemisphere. Both PICAs could not be visualized in the MR angiography, most likely due to the bilateral occlusion of the vessels. The vertebral artery showed an inconspicuous topographic course on both sides, without any signs of occlusion (Figure 1(e)).

On day two after stroke, the patient was transferred to the neurosurgical department due to incessant vomiting, progressive dysphagia, and a decreased level of consciousness.
Accordingly the GCS dropped from 15 to 13 . Cranial CT revealed an ischemic cerebellar edema with compression of the fourth ventricle and consecutive occlusive hydrocephalus. After the insertion of an external ventricular drainage (EVD) in the anterior horn of the right ventricle, the patient underwent urgent suboccipital decompressive craniectomy. The surgery was performed in prone position. A paramedian right-sided suboccipital craniectomy-crossing the midline to the contralateral side-was performed, combined with a partial $\mathrm{C} 1$ laminectomy. After the opening of the dura, clearly infarcted cerebellar tissue was removed until the space occupying effect was relieved. This procedure allowed a good visualization of the thrombosed bilateral PICA segments (Figure 2).

After about two weeks of disabling vertigo the patient was mobilized and had an otherwise uneventful recovery. At discharge the patient had a GCS of 15 and the reaction level 


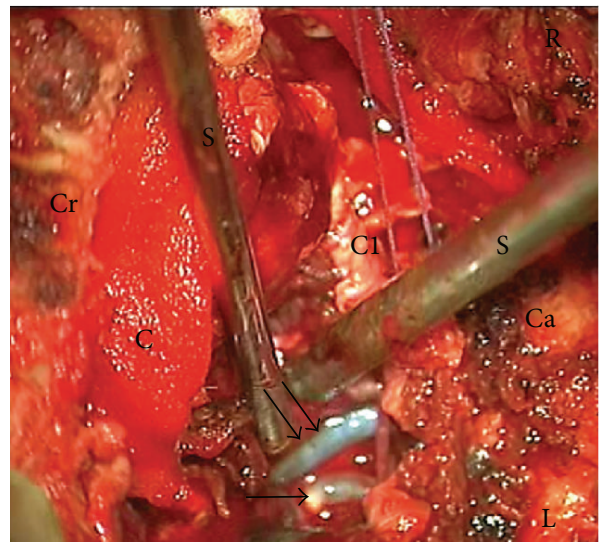

(a)

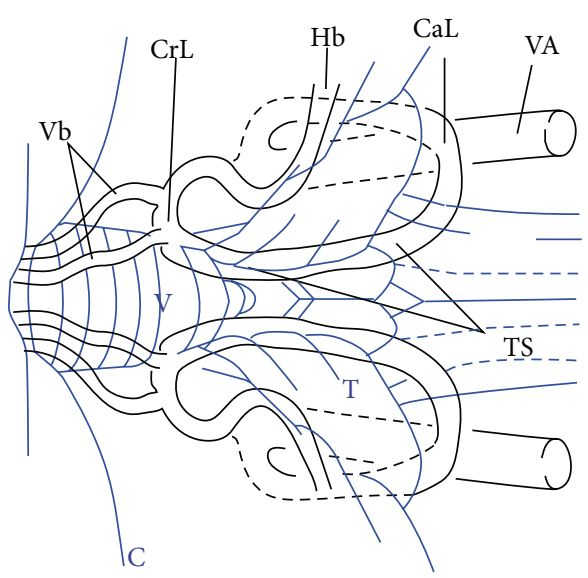

(b)

FIGURE 2: (a) Intraoperative photograph, showing readily visible bilateral PICAs, after the removal of the tonsils. The arteries were pulseless, rigid, and dark. A seemingly calcified thrombus was visible in left PICA (arrow). The right thrombosed PICA was bluish (double arrow). $\mathrm{C}$ indicates cottonoid placed over the remaining occipital bone; $\mathrm{Cl}$ : remaining parts of the $\mathrm{Cl}$ arch; Cr: cranial; Ca: caudal; L: left; R: right; S: suction. (b) Schematic drawing of the PICA. C indicates cerebellum; CaL: caudal loop of the PICA; CrL: cranial loop of the PICA; Hb: hemispheric branch; T: cerebellar tonsils, Ts: telovelomedullary segment; V: vermis; VA: vertebral artery; Vb: vermian branches.

scale score was 2. On late follow-up two months following stroke, the patient had a Modified Ranking Scale score of 2.

\section{Discussion}

As the PICA usually originates directly from a single vertebral artery, bilateral infarcts in the PICA distribution are rare. The clinical presentation is similar to that of unilateral PICA infarction [4]. Early diagnosis of cerebellar stroke with appropriate neurological examination, awareness of cerebellar stroke symptoms, and knowledge of brain imaging is crucial, as mortality is as high as $40 \%$ in misdiagnosed cerebellar infarcts [5]. The pathogenesis of bilateral PICA distribution infarction has not been sufficiently defined to date [4]. The most frequently postulated mechanism is that a dominant PICA gives rise to both medial branches and their occlusion is due to an embolus or atherosclerotic occlusion in the context of cerebrovascular disease $[4,6-8]$.

Given the rarity of bilateral PICA thrombosis with subsequent downstream cerebellar infarction, optimal medical and surgical management remains controversial. For embolic occlusion, thrombolysis in the hyperacute phase may be reasonable. At the time of admission our patient had a stroke onset more than 48 hours previously, so thrombolysis was not considered. Few long-term follow-up data are available regarding bilateral cerebellar infarction of PICA distribution. Overall outcome seems to be favorable in this stroke subgroup without primary brainstem involvement. Nonetheless, a bilateral cerebellar infarction may lead to progressive swelling, with brainstem compression and occlusive hydrocephalus. In this case, surgical decompression is warranted.

Pfefferkorn et al. observed no difference in outcomes between patients treated with suboccipital decompressive craniectomy for unilateral cerebellar infarction and those treated for bilateral cerebellar infarction, assuming that an infarction with a bilateral distribution should not be considered a contraindication for surgical decompression [9]. Further, early and aggressive treatment of bilateral cerebellar infarction with a suboccipital decompressive craniectomy resulted in favorable clinical outcome, especially when there was no established brainstem infarction [10]. Chen et al. included, in their series of 11 patients treated with decompressive suboccipital craniectomy and temporary ventriculostomy in malignant cerebellar edema after a large infarction, a total of three bilateral cerebellar infarctions. Since neurological improvement could be seen in most patients soon after the first postoperative day, they concluded that this operative procedure may be lifesaving in cases of progressive neurological deterioration while being on medical therapy for large infarctions [11].

Since its first description in 1994 by Tada et al. [8], there has been an increasing number reporting about acute bilateral cerebellar infarctions in the territory of both PICAs. However, this kind of stroke remains still rare. To the authors' knowledge, fewer than forty cases have been reported in the literature so far $[4,6-8,11-15]$, and only a fraction of it has been managed operatively. So far we are the first to visualize bilateral thrombosed PICAs in the televelomedullary segment. The ischemic stroke in our case was most likely attributable to a double, simultaneous embolic vessel occlusion by known cerebrovascular risk factors and visible thrombi. Because of the deteriorating clinical presentation, with progressive cerebellar swelling and a consecutive occlusive hydrocephalus, an immediate treatment of hydrocephalus with an EVD and surgical brainstem decompression by a suboccipital decompressive craniectomy with removal of necrotic cerebellar tonsils was performed. The PICA was exposed along the entirety of the according segment. Thus surgical access, which is readily obtained after sacrifice of the cerebellar tonsils, may offer surgical therapeutic options in hyperacute PICA occlusion. This potentially allows for 
microsurgical emergency procedures in case of failure of endovascular revascularisation or concomitance of contraindications in hyperacute PICA occlusion. These surgical revascularizations may include open thromb-/embolectomy [16], occipital artery-PICA end-to-side anastomosis [17, 18], and/or PICA/PICA side-to-side anastomosis [19] depending on the location and extension of thromboembolic occlusion. In case of vital cerebellar tonsils the PICA's televelomedullary segment may be approached by dissection of the cerebellomedullary fissure. Although emergency bypass procedures or embolectomy has been described for anterior circulation stroke $[16,20]$, the applicability for cerebellar stroke is certainly questionable and remains to be elucidated.

\section{Conflict of Interests}

The authors declare that there is no conflict of interests regarding the publication of this paper.

\section{References}

[1] E. Kumral, A. Kisabay, C. Ataç, C. Çalli, and N. Yünten, "Spectrum of the posterior inferior cerebellar artery territory infarcts: clinical-diffusion-weighted imaging correlates," Cerebrovascular Diseases, vol. 20, no. 5, pp. 370-380, 2005.

[2] C. S. Kase, B. Norrving, S. R. Levine et al., "Cerebellar infarction: clinical and anatomic observations in 66 cases," Stroke, vol. 24, no. 1, pp. 76-83, 1993.

[3] J. R. Lister, A. L. Rhoton Jr., T. Matsushima, and D. A. Peace, "Microsurgical anatomy of the posterior inferior cerebellar artery," Neurosurgery, vol. 10, no. 2, pp. 170-199, 1982.

[4] D. W. Kang, S. H. Lee, H. J. Bae, M. H. Han, B. W. Yoon, and J. K. Roh, "Acute bilateral cerebellar infarcts in the territory of posterior inferior cerebellar artery," Neurology, vol. 55, no. 4, pp. 582-584, 2000.

[5] S. I. Savitz, L. R. Caplan, and J. A. Edlow, "Pitfalls in the diagnosis of cerebellar infarction," Academic Emergency Medicine, vol. 14, no. 1, pp. 63-68, 2007.

[6] L. Brusa, M. Iannilli, G. Bruno et al., "Bilateral simultaneous cerebellar infarction in the medial branches of the posterior inferior cerebellar artery territories," Neurological Sciences, vol. 17, no. 6, pp. 433-436, 1996.

[7] S. W. Han, G. C. Cho, J. S. Baik, J. H. Park, J. Y. Kim, and J. H. Heo, "Bilateral cerebellar infarction caused by dominant medial posterior inferior cerebellar artery," Neurology, vol. 66, no. 7, pp. 1125-1126, 2006.

[8] Y. Tada, T. Mizutani, T. Nishimura, M. Tamura, and N. Mori, "Acute bilateral cerebellar infarction in the territory of the medial branches of posterior inferior cerebellar arteries," Stroke, vol. 25 , no. 3, pp. 686-688, 1994.

[9] T. Pfefferkorn, U. Eppinger, J. Linn et al., "Long-term outcome after suboccipital decompressive craniectomy for malignant cerebellar infarction," Stroke, vol. 40, no. 9, pp. 3045-3050, 2009.

[10] P. P. Tsitsopoulos, L. Tobieson, P. Enblad, and N. Marklund, "Clinical outcome following surgical treatment for bilateral cerebellar infarction," Acta Neurologica Scandinavica, vol. 123, no. 5, pp. 345-351, 2011.

[11] H. J. Chen, T. C. Lee, and C. P. Wei, "Treatment of cerebellar infarction by decompressive suboccipital craniectomy," Stroke, vol. 23, no. 7, pp. 957-961, 1992.
[12] C. Y. Hsieh, J. S. Lee, C. Y. Yu, and C. H. Chen, "Bilateral cerebellar infarction in the medial branches of posterior inferior cerebellar arterial territory-using endoscopic third ventriculostomy to relieve acute hydrocephalus," Clinical Neurology and Neurosurgery, vol. 110, no. 1, pp. 71-74, 2008.

[13] G. Gurer, G. Sahin, S. Cekirge, E. Tan, and O. Saribas, "Acute bilateral cerebellar infarction in the territory of the medial branches of posterior inferior cerebellar arteries," Clinical Neurology and Neurosurgery, vol. 103, no. 3, pp. 194-196, 2001.

[14] E. J. Sorenson, E. F. M. Wijdicks, K. R. Thielen, and T. M. Cheng, "Acute bilateral infarcts of the posterior inferior cerebellar artery," Journal of Neuroimaging, vol. 7, no. 4, pp. 250-251, 1997.

[15] G. Umashankar, V. Gupta, and S. I. Harik, "Acute bilateral inferior cerebellar infarction in a patient with neurosyphilis," Archives of Neurology, vol. 61, no. 6, pp. 953-956, 2004.

[16] T. Horiuchi, J. Nitta, K. Sakai, Y. Tanaka, and K. Hongo, "Emergency embolectomy for treatment of acute middle cerebral artery occlusion," Journal of Neurosurgery, vol. 106, no. 2, pp. 257-262, 2007.

[17] T. Inoue, A. Tamura, K. Tsutsumi, I. Saito, and N. Saito, "Acute to subacute surgical revascularization for progressing stroke in atherosclerotic vertebrobasilar occlusion," Acta Neurochirurgica, vol. 154, no. 8, pp. 1455-1461, 2012.

[18] R. M. Starke, M. Chwajol, D. Lefton, C. Sen, A. Berenstein, and D. J. Langer, "Occipital artery-to-posterior inferior cerebellar artery bypass for treatment of bilateral vertebral artery occlusion: The role of quantitative magnetic resonance angiography noninvasive optimal vessel analysis: Technical case report," Neurosurgery, vol. 64, no. 4, pp. E779-E781, 2009.

[19] M. Korja, C. Sen, and D. Langer, "Operative nuances of side-toside in situ posterior inferior cerebellar artery-posterior inferior cerebellar artery bypass procedure," Neurosurgery, vol. 67, no. 2, pp. ons471-ons477, 2010.

[20] E. S. Nussbaum, T. M. Janjua, A. Defillo, J. L. Lowary, and L. A. Nussbaum, "Emergency extracranial-intracranial bypass surgery for acute ischemic stroke," Journal of Neurosurgery, vol. 112 , no. 3, pp. 666-673, 2010. 


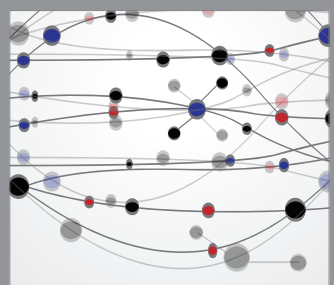

The Scientific World Journal
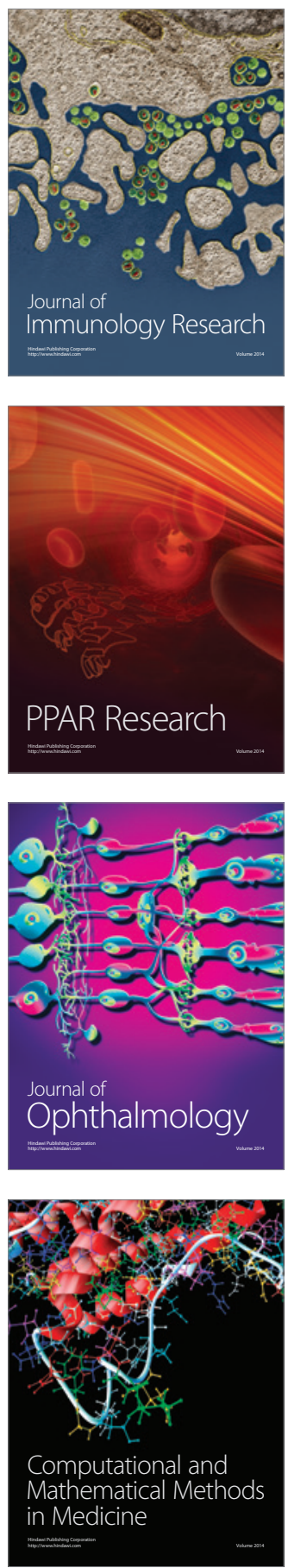

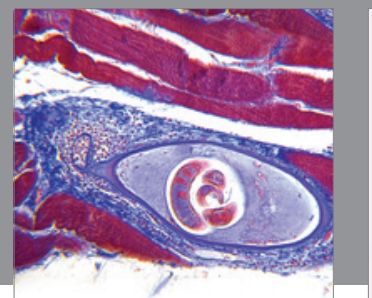

Gastroenterology

Research and Practice
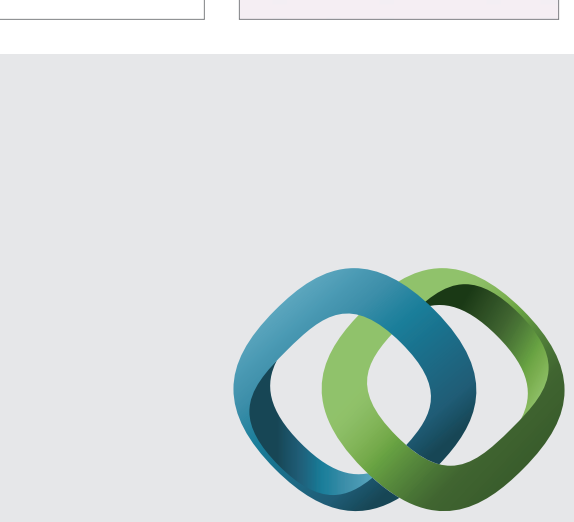

\section{Hindawi}

Submit your manuscripts at

http://www.hindawi.com
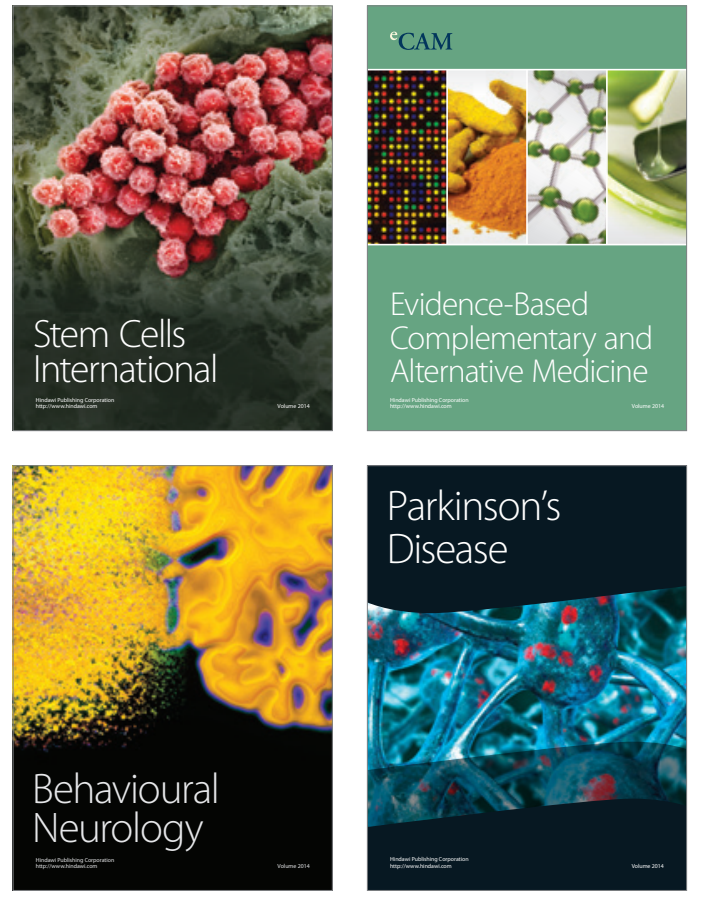
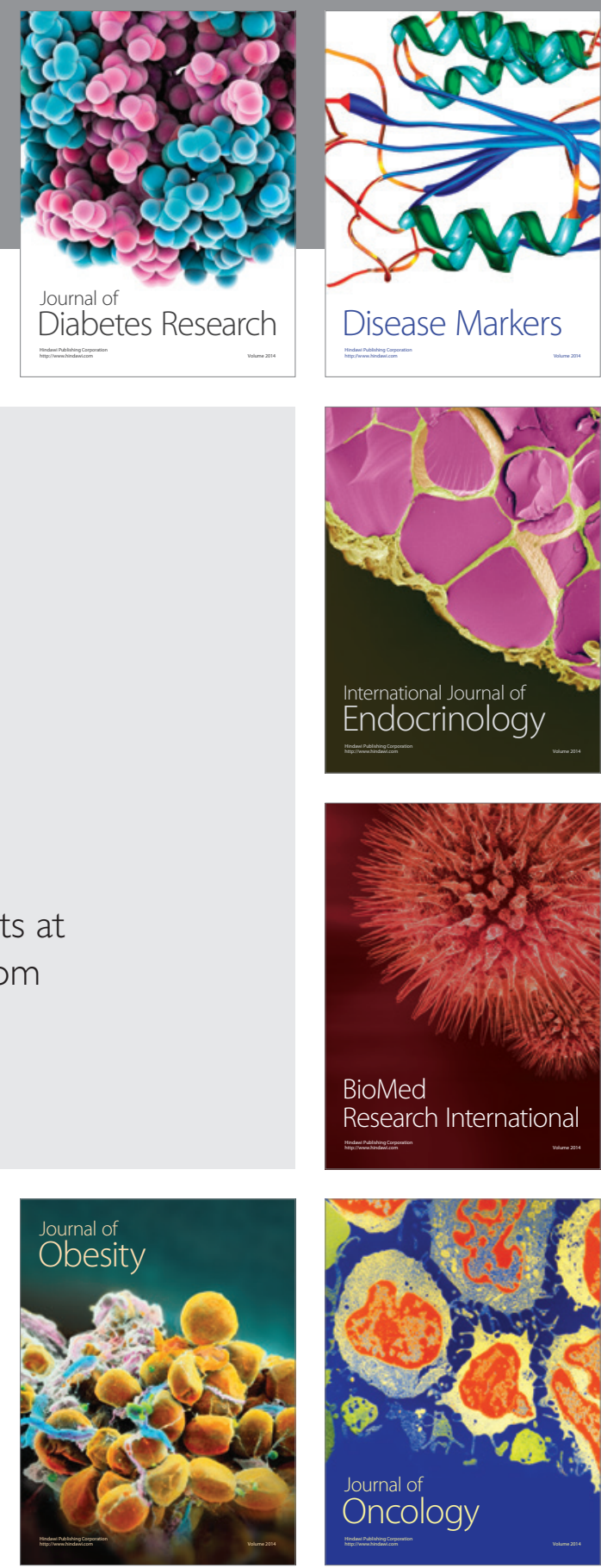

Disease Markers
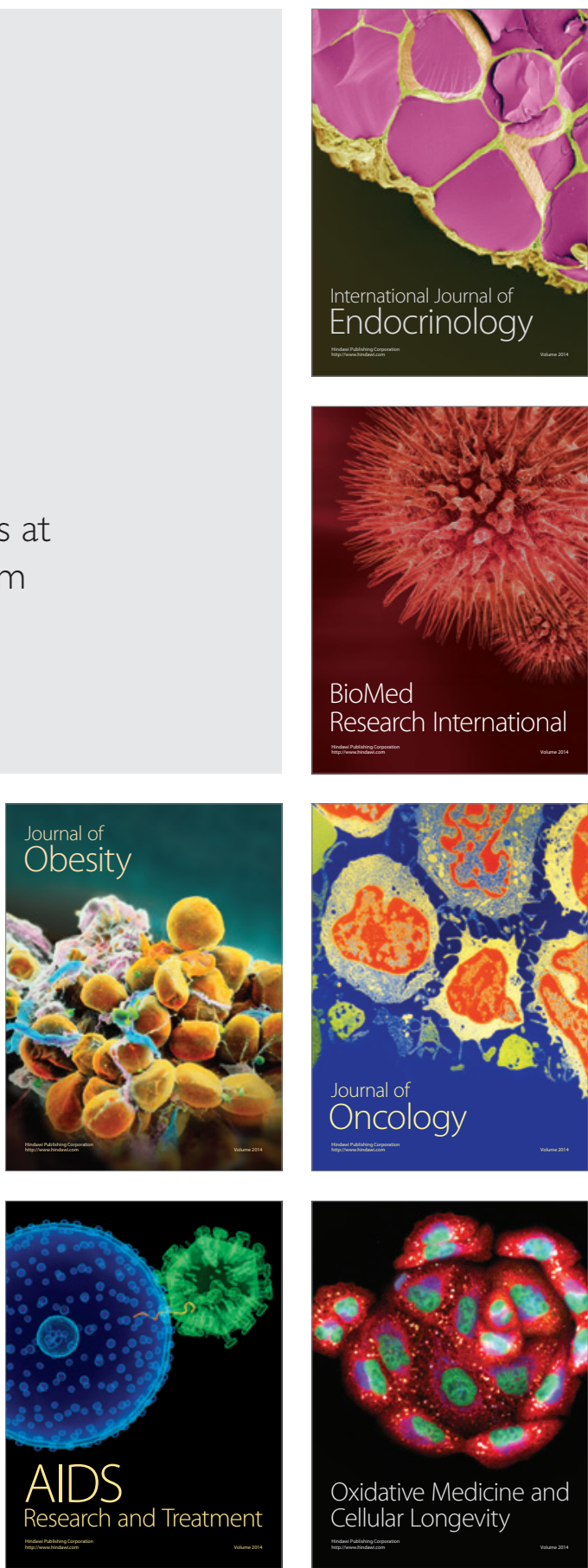